HUANG YAN, Ph.D. candidate

E-mail: tjyh@tongji.edu.cn

XIAONING ZHANG, Ph.D. ${ }^{1}$

(Corresponding author)

E-mail: cexzhang@tongji.edu.cn

XIAOLEI WANG, Ph.D. ${ }^{1}$

E-mail: xiaoleiwang@tongji.edu.cn

${ }^{1}$ School of Economics and Management

Tongji University

Shanghai 200092, China
Traffic Management Original Scientific Paper Submitted: 8 Apr. 2020 Accepted: 25 Sep. 2020

\title{
HIERARCHICAL PASSENGER HUB LOCATION PROBLEM IN A MEGAREGION AREA CONSIDERING SERVICE AVAILABILITY
}

\begin{abstract}
The rapid growth of the intercity travel demand has resulted in enormous pressure on the passenger transportation network in a megaregion area. Optimally locating hubs and allocating demands to hubs influence the effectiveness of a passenger transportation network. This study develops a hierarchical passenger hub location model considering the service availability of hierarchical hubs. A mixed integer linear programming formulation was developed to minimize the total cost of hub operation and transportation for multiple travel demands and determine the proportion of passengers that access hubs at each level. This model was implemented for the Wuhan metropolitan area in four different scenarios to illustrate the applicability of the model. Then, a sensitivity analysis was performed to assess the impact of changing key parameters on the model results. The results are compared to those of traditional models, and the findings demonstrate the importance of considering hub choice behavior in demand allocation.
\end{abstract}

\section{KEYWORDS}

hierarchical passenger hub location; megaregion area; service availability; hub choice behavior.

\section{INTRODUCTION}

Megaregions, which are highly integrated clusters of cities, gradually form in the process of urbanization. In China, approximately three-quarters (74\%) of the population is concentrated in nineteen current or emerging megaregions, and an additional 200 million migrants will move to cities by 2030. Thus, there is a pressing need for government agencies to establish a more cost-effective passenger transportation network to accommodate the fast-growing intercity/interregional travel demand. For example, an estimated 400 million intercity trips were taken from Shanghai in 2017, a 30\% increase from the number taken in 2013. Moreover, the trips within the Yangtze River Delta region account for $70 \%$ of those intercity trips from Shanghai. Unlike an urban passenger transportation network, which only meets the needs of intracity travel, a passenger transportation network on the megaregional scale is required to serve all levels of travel demand, such as area-level, regional, and national travel. Furthermore, due to the competitive environment in the passenger transportation market, passengers have a choice to select the best services. Hence, the Chinese government has formulated a series of policies for constructing and developing a hierarchical passenger transportation network to satisfy the multiple demands of passengers. As the key facilities in passenger transportation networks, passenger transportation hubs serve as switching and distribution points for megaregional travel from a set of origins to a set of destinations. Therefore, well-located passenger hubs are vital to the effectiveness of a hierarchical passenger transportation network.

Hierarchy is a significant characteristic of passenger transportation networks since each level of passenger transportation networks may provide different services to meet the demand at different geographical scales. From a supply-side perspective, each transportation mode has an optimal service range for market coverage. For example, Wang et al. [1] found that air transportation in China has achieved a dominant position in intercity passenger markets with average travel distance above 1280 $\mathrm{km}$. However, the entry of high-speed rail leads 
to a reduction in air passenger volumes [2]. What is more, rail transportation is considered the most competitive public transportation mode for the short-to-medium distance passenger market. The spatial overlap of the service range leads to competition between the two transportation modes. On routes of approximately $1000 \mathrm{~km}$ and above, air transportation can provide services that are available in rail transportation in a more efficient manner (e.g., travel time from hub to hub) but with a higher cost (e.g., ticket price) [3]. From a demand-side perspective, passenger needs can be satisfied by different levels of passenger hubs. People are more likely to choose the alternative with the highest attractiveness rather than being assigned to only one passenger hub. In previous studies on hub location problems (HLPs), most researchers simply assumed that each demand node receives services from the nearest hub. In contrast, the hub choice of passengers is based on a probability distribution, and passengers from different demand nodes will choose different hubs with a certain probability, which is related to the fare, travel time, distance, and even environmental concerns. For this reason, decisions regarding the location and level of hubs need to be carefully planned.

In this paper, we present a hierarchical passenger hub location model for megaregional passenger transportation network design. This model considers a hierarchical passenger transportation network for multilevel travel demands and the new service availability type, and a logit discrete-choice model is used to determine the proportion of passengers that access the passenger hub depending on its own utility. These considerations make the model realistic in terms of describing the real behaviors of passengers regarding the choice of passenger transportation hubs. The objective function involves the minimization of the total cost, including the access cost, time spent on transit, and investment in planning. To the best of our knowledge, this is the first paper that presents hub choice behaviors for a hierarchical passenger transportation network considering service availability at the megaregional scale. As a case study, the proposed model is implemented for the Wuhan metropolitan area (WHM) located in the eastern Hubei Province, Central China. The WHM comprises nine cities and 36 counties. It should be noted that the infrastructure investment cost and the hierarchical travel demand spatially vary because of regional differences in social and economic factors.
Different competitive degree among three transportation modes (intercity bus, railway, and air) are taken into account in four scenarios. Modelling results for the case study in different scenarios verify the validity of the proposed model.

This paper is organized as follows. Section 2 discusses the literature related to HLPs. In the third section, we describe a hierarchical passenger hub location model for a megaregion. Then, we introduce a case study involving the application of the model to the WHM and present the results of the proposed model. Finally, the conclusions and recommendations for future research directions are briefly discussed in the last section.

\section{LITERATURE REVIEW}

HLPs have been successfully applied in many subjects. Pašagić-Škrinjar et al. [4] described the location problems of the city terminals on the logistic network as hub location problems. Kim and Soh [5] developed a P-median hub location model to determine optimal locations of school bus stops. Šarac et al. [6] formulated a set covering location model on the postal network of the public postal operator in Serbia. Other examples of HLPs may include telecommunication systems, computer networks, and emergency services.

Research on HLPs involving transportation networks began with the pioneering work of O'Kelly [7]. The first quadratic model for HLPs in an airline network was presented by O'Kelly [8]. Since then, there have been different types of HLPs. Campbell [9] formulated a P-hub median model considering different allocation mechanisms: single allocation and multiple allocation. Moreover, he also presented an integer programming formulation of uncapacitated hub location model [10]. Costa et al. [11] proposed a bi-criteria approach to the capacitated single allocation hub location problem. The comprehensive surveys of HLPs have been provided in the literature [12].

Numerous studies have focused on the hierarchical features of transportation networks. The first work on hierarchical hub location problems (HHLPs) was performed by Flynn and Ratick [13], who formulated a multi-objective model considering the hierarchy of airline service levels. Current [14] also considered two path levels (the primary and secondary path) in a hierarchical network under a minimum cost objective. O'Kelly and Lao [15] developed a linear programming model for HHLPs 
with two hubs: one master hub that was connected with all demand nodes and one mini-hub that only served as a regional sorting center; moreover, this was the first study to introduce the mode choice concept in HLPs. In another work [16], an intermodal hub location approach was developed and both railway and road transportation between nodes were considered to minimize the total transportation costs. Yu et al. [17] proposed a cluster-based hierarchical location model for three-level transit hubs (region level, area level and local level) to minimize the demand-weighted total travel time. These studies focused on the location hubs at each level as well as the allocation of demand nodes to these hubs.

One of the most important aspects of HHLPs is considering the service availability of hierarchical hubs, which encompasses the relationships among various levels of hierarchy. There are two basic types of service availability: nested hierarchy and non-nested hierarchy. In a nested hierarchy, a higher-level hub provides all the services available at a lower-level hub. In a non-nested hierarchy, hubs at each level offer different services [18]. The level of the transportation hub determines the coverage distance and the service type provided by the hub. Most transportation systems are organized based on nested service availability. Karimi et al. [19] designed a capacitated hub median location problem with a nested hierarchical structure and used a real-world dataset from the hub location problem corresponding to the Iranian hub airport location. $\mathrm{Li}$ et al. [20] described a hierarchical service network with a nested structure and investigated a multi-period hierarchical location problem for a transportation hub in an urban agglomeration area. Torkestani et al. [21] designed nested and non-nested networks in a capacitated hierarchical multimodal hub location problem considering multiple periods in the planning horizon.

Another important aspect of HHLPs that has been traditionally overlooked is the allocation strategy, resulting in the demand nodes needing to be allocated to the hub nodes by the system designers. In practice, however, passengers at a demand node can choose different hub nodes within a reasonable range according to their preferences. Hence, the hub choice behavior of passengers is considered in this study. Debrezion et al. [22] developed a nested logit model to explain choice behaviors concerning departure stations and access modes for Dutch passengers. Wang and $\mathrm{Qu}$ [23] investigated how commuters choose stations based on the generalized cost considering the availability of seats. Discrete-choice models are typically used to formulate facility choice behaviors. There are two important factors that affect hub choice: the service availability of the hub and the accessibility of the hub. The hierarchical services provided at hub nodes correspond to the multilevel travel demand of passengers. Different from prior studies, this paper focuses on the effects of hub choice behavior on the hierarchical passenger hub location problem in a megaregion area.

\section{PROBLEM FORMULATION}

The HLPs that we study in this paper focus on determining the locations of passenger hubs and designing a hierarchical passenger hub network in a megaregion area. The location decision involves the selection of a set of nodes to establish passenger hubs, whereas the passenger hub network design decisions are made at the level of each passenger hub. The optimal allocation of the travel demand through the network is also determined.

\subsection{Hierarchical passenger hub structure}

In this section, we propose a hierarchical passenger hub structure in a megaregion area. In practice, a public transportation network is required to meet the multiple demands of passengers. Any single transportation mode cannot be capable of covering all geographical scales of the demand because of its limited service area. Thus, the hierarchy of transportation hubs can be categorized into $s$ levels (where level 1 is the lowest level and level s is the highest hierarchical level) depending on the service range of market coverage. We know that passengers could access different levels of hubs that provide the s-level services required. In this way, the s-level demand is provided by hubs of the same level that are non-nested hubs or by hubs of same level or higher levels in a nested hierarchy. However, the service availability of a megaregional hub network is neither nested nor non-nested. Considering a four-level hierarchical passenger hub network (Figure 1), the service area provided by hubs at each level is different, but there is an overlapping service range between hubs at two adjacent levels, which results in spatial 'competition' between transportation modes. Therefore, the service range is classified into two categories: (1) noncompetitive areas where the 


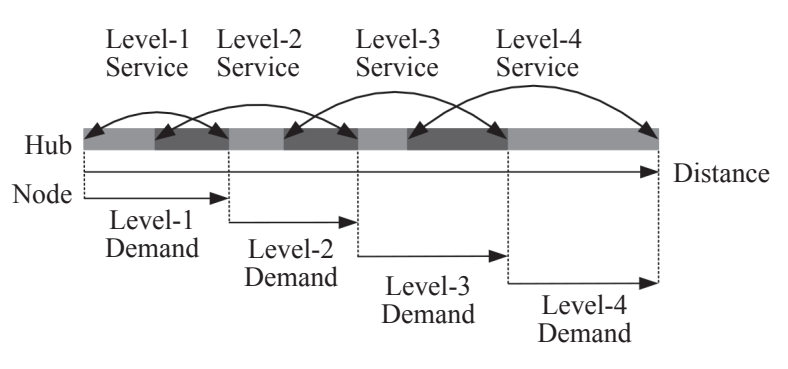

Noncompetitive Area Competitive Area

Figure 1 - Hierarchical Passenger hub structure in a megaregional area

travel demand can be served by hubs only at one level and (2) competitive areas where the travel demand can be served by hubs at the same level or higher levels.

To provide a detailed description of this passenger hub structure, we define $S=\{1,2, \ldots, s\}$ to denote the level of passenger hubs. Let $\left(r_{k}^{o}, r_{k}^{d}\right)$ be the service range of a level- $k(k \in S)$ hub. Thus, the noncompetitive area of the level- $k$ hub can be represented as $\left(r_{k-1}^{d}, r_{k+1}^{o}\right)$ for $1<k<s\left(\left(0, r_{1}^{g}\right)\right.$ for $k=1$, and $\left(r_{s-1}^{d}, r_{s}^{d}\right)$ for $k=s)$, and service competition between a level- $k$ hub and a level-k+1 hub may occur in the competitive area $\left(r_{k+1}^{o}, r_{k}^{d}\right)$ for $1 \leq k<s$. The parameter $\lambda_{k}$ is used to measure the degree of competition between transportation modes, which can be expressed as:

$\lambda_{k}=\left\{\begin{array}{cc}\frac{r_{k}^{d}-r_{k+1}^{o}}{r_{k}^{d}-r_{k}^{o}} & 1 \leq k<s \\ 0 & k=s\end{array}\right.$

It should be noted that the service availability of the level- $k$ hub would be nested when $\lambda_{k}=1$ $\left(r_{k}^{o=}=r_{k+1}^{o}\right)$. Similarly, if we set $\lambda_{k}$ to $0\left(r_{k}^{d=r_{k+1}^{o}}\right)$, the hub network would have a non-nested hierarchical structure because the level- $k$ hubs provide a distinct service only for the level- $k$ travel demand.

\subsection{Hub choice model}

Let $I=\{1,2, \ldots,|I|\}$ be the set of demand nodes and $H=\{1,2, \ldots, s\}$ be the set of travel demand levels. $w_{i h}$ denotes the amount of level- $h(h \in H)$ demand at node $i \in I$. To make the problem tractable, we define $\left(r_{h-1}^{d}, r_{h}^{d}\right)$ to denote the range of the level- $h$ travel demand $\left(\left(0, r_{1}^{d}\right)\right.$ for $\left.h=1\right)$, which encompasses a noncompetitive area and a competitive area. We note here that the existence of a competitive area requires the definition of additional variables because demand variables are aggregate variables and because $w_{i h}$ does not reflect whether the range of the demand is within a competitive area. Accordingly, we index $a \in A=\{C, N C\}$ to denote the travel demand type. The travel demands are then accounted for as one of two types: $w_{i h}^{C}$ is the level- $h$ demand within a competitive area originating at node $i$ that can be met by passenger hubs at the same level or higher levels, and $w_{i h}^{N C}$ is the demand that must be met by same-level passenger hubs.

Transportation hubs can be established among a set of candidate locations, $J=\{1,2, \ldots,|J|\}$, to serve passengers. Opening a level- $k$ hub requires a basic $\operatorname{cost} f_{k}$ of operation. However, the infrastructure investment cost spatially varies because of regional differences. For example, the construction cost of passenger hubs is closely related to the land price, which is different in each city. We define $\beta_{j}$ to denote the cost difference at hub $j \in J$. We also define the binary location variable $x_{j k}$, which assumes a value of one if a level- $k$ hub is located at $j \in J$ and zero otherwise. Therefore, the total transportation hub operation cost is $\sum_{j \in J} \sum_{k \in S} x_{j k} f_{k} \beta_{j}$.

As noted above, hubs at each level will attract passengers with different travel demands. A passenger within a competitive area can choose a same-level or higher-level passenger hub service. The other type of travel demand can only be met by same-level hubs. For clarity, the hub choice set $H_{i h}^{a}$ for the level- $h$ demand originating at node $i$ is separately defined according to the travel demand type $a \in A$.

$H_{i h}^{C}=\left\{j \in J \mid \sum_{h \leq k \leq h+1} x_{j k}=1\right\}:$ the hub choice set for the level- $h$ demand within a competitive area originating at node $i$.

$H_{i h}^{N C}=\left\{j \in J \mid x_{j h}=1\right\}:$ the hub choice set for the level- $h$ demand within a noncompetitive area originating at node $i$.

In addition, we assume that passengers only select a service when they are within a reasonable distance from a hub. Passengers with high-level demands may accept longer access distances. Thus, let $R_{h}$ be the maximum acceptable distance for the level- $h$ demand in hub selection. By doing so, we can obtain a specific and feasible hub set for formulating the hub choice model to estimate the allocation of the travel demand.

Before introducing demand allocation, we assume that the travel demand at each node unaffected by any other factors, e.g., the travel time, which means that the travel demand at each node is inelastic. We also assume that passengers are rational, which means that the hub choices of passengers depend on the perceived utility of probable hubs. However, for each 
hub, the utility reflects the passengers' preferences regarding the transportation cost and total travel time.

$C_{i h j}^{a}$ denotes the transportation cost associated with the level- $h$ travel demand from node $i$ to hub $j$. The transportation cost $C_{i h j}^{a}$ is composed of two components: (1) the travel cost from a node to a passenger hub and (2) the fare charged by hub service providers, which reasonably increases with the service level. We use $c_{0}$ to denote the cost of transportation per unit distance by arcs between nodes and passenger hubs. Let $c_{k}$ be the unit cost per unit distance for a level- $k$ service. The transportation cost can be calculated as follows.

$$
C_{i h j}^{a}=c_{0} d_{i j}+c_{k} d_{h}^{a} \quad \forall a \in A, i \in I, j \in J, h \in H
$$

where $d_{i j}$ is the distance between demand node $\mathrm{i}$ and potential passenger hub node $j$ and $d_{h}^{a}$ is the travel distance for the level- $h$ demand. Note that $d_{h}^{a}$ is sampled from a range of level- $h$ travel distances in practice.

We also denote the total travel time associated with the level- $h$ travel demand from node $i$ for services at passenger hub $j$ by $T_{i h j}^{u}$, which consists of two components: (1) the access time from node $i$ to hub $j$ denoted by $t_{i j}$ and (2) the travel time from hub to hub defined by $d_{h}^{a} / V_{k}$. In this case, $V_{k}$ is the travel speed of the level- $k$ service; that is,

$$
T_{i h j}^{a}=t_{i j}+\frac{d_{h}^{a}}{V_{k}} \quad \forall a \in A, i \in I, j \in J, h \in H
$$

From the above relations, $U_{i h j}^{a}$ is defined as the random utility of hub choice representing the preference of passengers on hub $j$, and $V_{i h j}^{a}$ is the certainties for $U_{i h j}^{a}$

$$
\begin{aligned}
U_{i h j}^{a} & =-C_{i h j}^{a}-\delta_{i} T_{i h j}^{a}+\varepsilon_{i h j}^{a} \\
& =V_{i h j}^{a}+\varepsilon_{i h j}^{a} \quad \forall a \in A, i \in I, j \in J, h \in H
\end{aligned}
$$

where $\delta_{i}$ represents the value of time (VOT) of passengers at node $i . \varepsilon_{i h j}^{a}$ is the stochastic term of $U_{i h j}^{a}$, which is assumed to be independent, identically distributed, and random following the Gumbel distribution with a mean of zero.

Given a set of hub locations, the demand is allocated to hubs according to the corresponding utility. Let $y_{i h j}^{a}$ be the fraction of the level- $h$ demand at node $i$ for passengers who choose services from hub $j \in H_{i h}^{a}$. We select the logit-based discrete-choice model to identify passengers' decisions regarding hub service. Hence, the demand allocations can be calculated as follows:

$$
y_{i h j}^{a}=\frac{\exp \left(\theta V_{i h j}^{a}\right)}{\sum_{e \in H_{i h}^{a}} \exp \left(\theta V_{i h e}^{a}\right)} \quad \forall a \in A, i \in I, j \in J, h \in H
$$

where $\theta$ is the scale parameter related to the variance of the stochastic term $\varepsilon_{i h j}^{a}$ which complies with Gumbel distribution: $F\left(\varepsilon_{i h j}^{a}\right)=e^{-e^{-\theta \varepsilon_{i h j}^{a}}}$. A high $\theta$ value indicates a smaller variance of the stochastic term, which means passengers prefer to choose hub with high certain utility.

\subsection{Model formulation}

In this section, we formulate a hierarchical passenger hub location model for minimizing the total cost, which includes the hub operation cost and transportation cost associated with the travel demand. Furthermore, we consider different capacities for hubs at each level and define two parameters $Q_{k}$ and $q_{k}$ that represent the maximum and minimum capacities for a level- $k$ hub, respectively.

With the notation above, the mathematical formulation of the model is as follows:

$$
\begin{aligned}
& \operatorname{Min} \sum_{j \in J} \sum_{k \in S} x_{j k} f_{k} \beta_{j}+ \\
& +\sum_{i \in I} \sum_{h \in H} \sum_{a \in A} \sum_{j \in J} y_{i h j}^{a} w_{i h}^{a}\left(C_{i h j}^{a}+\delta_{i} T_{i h j}^{a}\right)
\end{aligned}
$$

subject to

$$
\begin{aligned}
& \sum_{k \in S} x_{j k} \leq 1 \quad \forall j \in J \\
& \sum_{i \in I} \sum_{h \in H} \sum_{a \in A} y_{i h j}^{a} w_{i h}^{a} \leq x_{j k} Q_{k} \quad \forall j \in J \\
& \sum_{i \in I} \sum_{h \in H} \sum_{a \in A} y_{i h j}^{a} w_{i h}^{a} \geq x_{j k} q_{k} \quad \forall j \in J \\
& Q_{k} \geq q_{k} \quad \forall k \in S \\
& \left(d_{i j}-R_{h}\right) y_{i h j}^{a} \leq 0 \quad \forall a \in A, i \in I, j \in J, h \in H \\
& x_{j k} \in\{0,1\} \quad \forall j \in J, k \in S \\
& y_{i h j}^{a} \geq 0 \quad \forall a \in A, i \in I, j \in J, h \in H
\end{aligned}
$$

The objective function 6 represents the total cost of hub operation and transportation for the corresponding travel demand. Constraints 7 guarantee that different levels of hubs cannot be located at the same node. Constraints 8 require the travel demand met by a hub not to exceed the maximum capacity. Constraints 9 ensure that hubs at each level can only be established at candidate locations with a demand higher than the minimum quantity. Constraints 10 prevent the contradiction between Constraints 8 and 9. Constraints 11 are used to prevent any fraction of the passengers 
with a level- $h$ demand at node $i$ from choosing hub $j$ when hub $j$ is outside of the allowable travel area. Finally, Constraints 12 and 13 are domain constraints.

The model described by 6-13 is an integer nonlinear programming model. The nonlinearity of this formulation is considered by the utilization of a logit model to determine the proportion of passengers at each passenger hub. Thus, we use the following equivalent set of constraints to linearize the logit model and obtain a mixed integer linear program.

$$
\begin{aligned}
& \sum_{j \in J} y_{i h j}^{a}=1 \quad \forall a \in A, i \in I, h \in H \\
& y_{i h j}^{C} \leq \sum_{h \leq k \leq h+1} x_{j k} \quad \forall i \in I, j \in J, h \in H \\
& y_{i h j}^{N C} \leq x_{j h} \quad \forall i \in I, j \in J, h \in H \\
& y_{i h j}^{C} \leq \frac{\exp \left(\theta V_{i h j}^{C}\right)}{\exp \left(\theta V_{i h e}^{C}\right)} y_{i h e}^{C}+\left(1-\sum_{h \leq k \leq h+1} x_{j k}\right) \\
& \forall i \in I, j, e \in J, h \in H \\
& y_{i h j}^{N C} \leq \frac{\exp \left(\theta V_{i h j}^{N C}\right)}{\exp \left(\theta V_{i h e}^{N C}\right)} y_{i h e}^{N C}+\left(1-x_{j h}\right) \\
& \forall i \in I, j, e \in J, h \in H
\end{aligned}
$$

Constraints 14 ensure that the sum of the fractions of the chosen facility is 1 . Constraints 15 and 16 stipulate that passengers can receive service only from passenger hubs located at the corresponding level. Finally, according to Aros-Vera et al. [24], the logit model in Equation 5 can be reformulated by Constraints 17 and 18 .

\section{CASE STUDIES}

In this section, we apply our model to a real-world case study. A set of experiments is conducted under different parameter settings to validate the developed model. All experiments are performed on an Intel(R) Core(TM)2 CPU running at $2.6 \mathrm{GHz}$ and with 16 GB of RAM and a 64-bit Windows 10 operating system. The free toolbox YALMIP R20190425 [25] together with MATLAB R2019a is adopted to model the example. The commercial solver Gurobi optimizer v8.1.1, as an MIP solver, is used to implement the presented model. The optimal solution is obtained in an acceptable computational time (less than $2 \mathrm{~h}$ for each scenario).

\subsection{Experimental results}

We present a practical case study based on the Wuhan metropolitan area, which is located in eastern Hubei Province in Central China, to illustrate the applicability of the proposed model. With an area of approximately $58,000 \mathrm{~km}^{2}$ and an estimated population of 32.1 million in 2018, WHM is the fifth largest metropolitan area in China. As shown in Figure 2, this area comprises 36 counties, including 9 cities (Qianjiang, Tianmen, Xiantao, Ezhou, Huangshi, Xianning, Xiaogan, Huanggang, and Wuhan). In addition, WHM is one of the most densely populated and fastest-growing areas in China. The rapid development in WHM has increased pressure on the intercity passenger transportation network. Hence, it is crucial to design a hierarchical passenger transportation network that satisfies the multiple demands of passengers.

In this paper, we consider three service types for intesrcity travel: intercity bus, railway, and air services. The competitive relationship between transportation modes has gone through several phases with the development of the passenger transportation market. Based on historical data and expert opinions, we design three scenarios, each of which specifies the effect of competition between transportation modes on the hierarchical passenger hub structure. It is assumed that the two competitive areas in scenario 1 exist in the $100-300 \mathrm{~km}$ range for the short-distance travel market and in the 400-1000 km range for the medium-distance travel market. However, the rapid construction of rail infrastructure expands the level-2 service range to $50-1500 \mathrm{~km}$ in scenario 2, resulting in increased competition within the market for intercity travel. Furthermore, as train speeds become faster, railway services are likely to impose significant competitive pressures on intercity bus and air transportation in the short- to medium-distance passenger markets. As a result, the service ranges of level-1 and level-3 services are reduced to short routes less than 200 $\mathrm{km}$ and long routes over $800 \mathrm{~km}$ in scenario 3, respectively. The parameter values for each scenario are shown in Table 1.

Based on the defined hierarchical passenger hub structure, passenger hubs at each level only offer one type of transportation mode in scenarios 1,2 , and 3. However, there has been increased interest in planning comprehensive passenger transportation hubs that integrate at least two transportation types [26]. For this purpose, we consider the hierarchical location of comprehensive passenger transportation hubs in the WHM. A new scenario is designed to analyze the effect of comprehensive passenger transportation hubs. Thus, in scenario 4 , the parameter values are the same as that in scenario 3 , but the 
$\underline{\text { Yan H, Zhang X, Wang X. Hierarchical Passenger Hub Location Problem in a Megaregion Area Considering Service Availability }}$

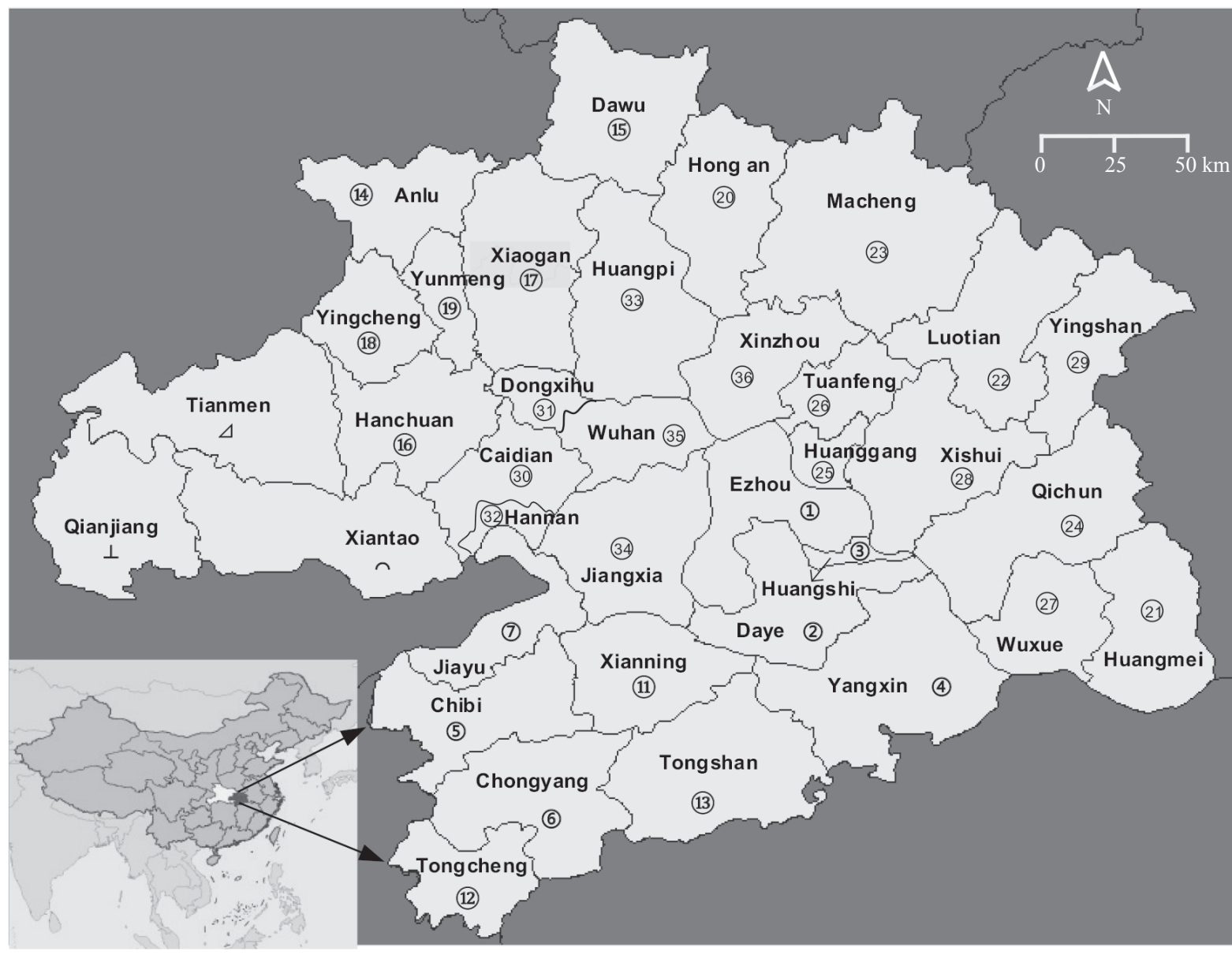

WHM

Qianjiang Tianmen Xiantao Ezhou Huangshi Xianning Xiaogan Huanggang Wuhan Cities

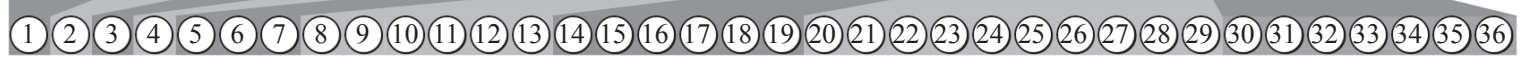

Figure 2 - Location of cities in the WHM

Table 1 - Parameter values in each scenario

\begin{tabular}{|c|c|c|c|c|}
\hline \multirow{2}{*}{\multicolumn{2}{|c|}{ Parameters }} & \multicolumn{3}{|c|}{ Scenario number } \\
\hline & & \multirow{2}{*}{$\frac{1}{(0,300)}$} & \multirow{2}{*}{$\frac{2}{(0,300)}$} & \multirow{2}{*}{$\frac{3}{(0,200)}$} \\
\hline \multirow{3}{*}{$\left(r_{k}^{o} r_{k}^{d}\right)[\mathrm{km}]$} & $k=1$ & & & \\
\hline & $k=2$ & $(100,1000)$ & $(50,1500)$ & $(50,1500)$ \\
\hline & $k=3$ & $(400, \sim)$ & $(400, \sim)$ & $(800, \sim)$ \\
\hline \multirow{3}{*}{$f_{k}\left(10^{6} \mathrm{CNY}\right.$ per day $)$} & $k=1$ & 5 & 5 & 5 \\
\hline & $k=2$ & 50 & 50 & 60 \\
\hline & $k=3$ & 80 & 80 & 80 \\
\hline \multirow{3}{*}{$Q_{k} / q_{k}\left(10^{4}\right.$ persons per day $)$} & $k=1$ & $8 / 1$ & $8 / 1$ & $8 / 1$ \\
\hline & $k=2$ & $25 / 3$ & $25 / 3$ & $30 / 5$ \\
\hline & $k=3$ & $15 / 3$ & $15 / 3$ & $15 / 3$ \\
\hline \multirow{3}{*}{$V_{k}[\mathrm{~km} / \mathrm{h}]$} & $k=1$ & 70 & 70 & 70 \\
\hline & $k=2$ & 100 & 100 & 200 \\
\hline & $k=3$ & 900 & 900 & 900 \\
\hline \multirow{3}{*}{$C_{k}[\mathrm{CNY} / \mathrm{km}]$} & $k=1$ & 0.32 & 0.32 & 0.32 \\
\hline & $k=2$ & 0.20 & 0.20 & 0.45 \\
\hline & $k=3$ & 0.68 & 0.68 & 0.68 \\
\hline
\end{tabular}


hubs at different levels can be located at the same node. Constraints 7 are replaced by the following constraints in this case:

$\sum_{k \in S} x_{j k} \leq 3 \quad \forall j \in J$

For the purpose of our study, each county is considered a demand node with different demand generation rates according to the population. The total demand in each county is constant. However, the demand at each level varies based on the range of travel distances. We estimate the VOT according to the wage rate of each county, which is the per capita GDP divided by the total work time in a year. The maximum acceptable distance for service at each level is $50 \mathrm{~km}, 100 \mathrm{~km}$, and $200 \mathrm{~km}$. The distance between two nodes is calculated based on the actual road distance between two county centers. We assume that passengers access the hub by public transportation (bus/tram/metro) or car according to the access distance. The costs of public transportation and cars are set at 0.2 Chinese Yuan (CNY) per kilometer and 0.6 CNY per kilometer, respectively. The access time is calculated according to the distance divided by velocity, and the values are set as $30 \mathrm{~km} / \mathrm{h}$ (by public transportation) and $50 \mathrm{~km} / \mathrm{h}$ (by car). The scale parameter in the logit-based discrete-choice model is set as -0.1 .

We investigate the impact of the hierarchical hub structure in four different scenarios. The experimental results are summarized in Table 2. Note that TC, OC, TRC, and AC represent the total cost, hub operation cost, travel cost, and access cost, respectively. The total demand met by hubs at each level is compared.

The expansion of the level-2 service range in scenario 2 leads to more passengers choosing level-2 hubs, and this mainly accounts for the reduction in the demand met by level 1 and level 3 hubs compared with that in scenario 1 . When the unit transportation cost of level-2 services increases in scenario 3 , the short-distance demand severed by level-2 hubs decreases although the travel speed of level-2 services doubles. Also, the total cost of level-2 services is much less than that of level-3 services, so almost all level-2 passengers within the competitive area choose level-2 hubs, and the total demand served by level-3 hubs remains unchanged in scenarios 2, 3, and 4. Note that the optimal locations of hubs at each level found for scenarios 1, 2 , and 3 are unchanged. The traveling cost and the access cost in scenario 2 are the lowest among all scenarios. This shows that more intense competition between transportation modes has a positive impact on passengers.

Furthermore, with the planning of comprehensive transportation hubs in scenario 4, the hub operation cost per day only decreased by $1.21 \%$ in comparison with that in scenario 3. However, this planning allows the transportation agency to save costs considerably in the entire life of the passenger hubs. The results show that nodes 13 and 24 are comprehensive transportation hubs that provide level-1 and level-2 services. Both level-1 and level-3 hubs are located at node 22. Figure 3 illustrates the

Table 2 - Experimental results in each scenario

\begin{tabular}{||c|c|c|c|c|c||}
\hline \multicolumn{2}{|c|}{ Scenario number } & 1 & 2 & 3 & 4 \\
\hline \hline \multirow{4}{*}{$\begin{array}{l}\text { Total demand served } \\
\text { by level-k hubs }\end{array}$} & $k=1$ & 308,567 & 289,609 & 299,445 & 294,470 \\
\cline { 2 - 6 } & $k=2$ & 304,952 & 543,024 & 533,188 & 538,163 \\
\cline { 2 - 6 } & $k=3$ & 262,937 & 43,823 & 43,823 & 43,823 \\
\hline \multirow{5}{*}{ Cost (CNY) } & $\mathrm{TC}$ & $563,267,750$ & $462,645,421$ & $552,205,325$ & $550,603,465$ \\
\cline { 2 - 6 } & $\mathrm{OC}$ & $195,106,800$ & $195,106,800$ & $214,879,400$ & $212,273,900$ \\
\cline { 2 - 6 } & $\mathrm{TRC}$ & $328,176,643$ & $235,467,330$ & $304,681,842$ & $304,615,959$ \\
\cline { 2 - 6 } & $\mathrm{AC}$ & $39,984,307$ & $32,071,291$ & $32,644,083$ & $33,713,606$ \\
\hline \multirow{5}{*}{ Optimal locations } & $k=1$ & $1,3,6,7,8,11,12$, & $1,3,6,7,8,11,12$, & $1,3,6,7,8,11,12$, & $1,3,7,8,12,13,15$, \\
& & 29,31 & $15,19,20,21,26$, & $15,19,20,21,26$, & $19,20,21,22,24$, \\
& $k=2$ & $2,13,24,33$ & 29,31 & 29,31 & 26,31 \\
\cline { 2 - 6 } & $k=3$ & 10,22 & $13,24,33$ & $2,13,24,33$ & $2,13,24,33$ \\
\hline \hline
\end{tabular}




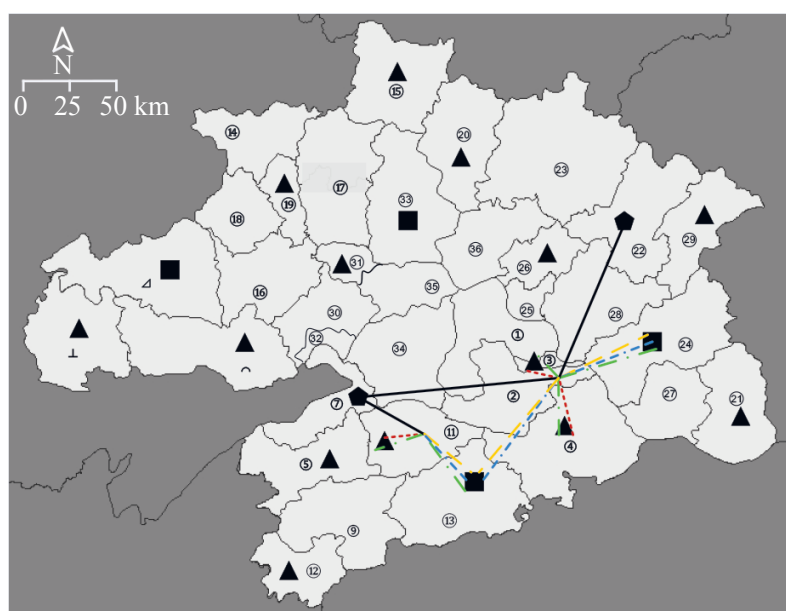

a) Scenario 3

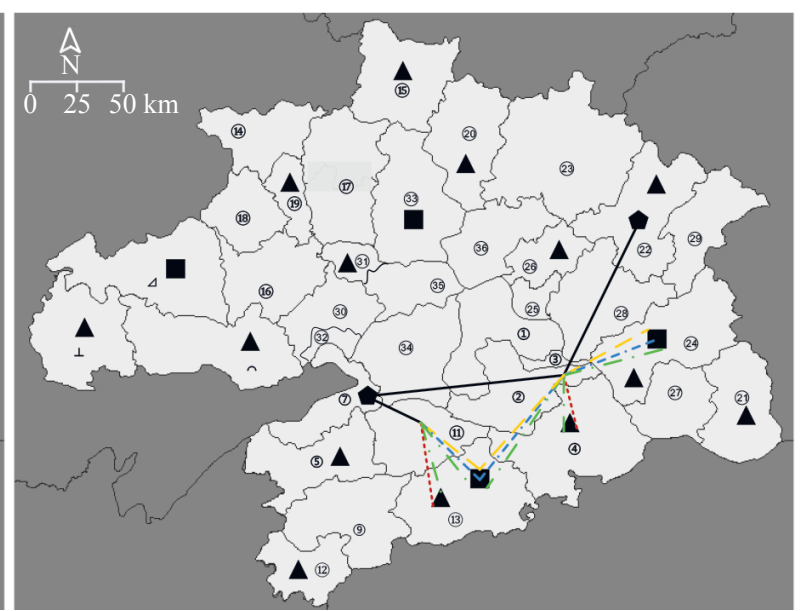

b) Scenario 4
$\Delta$

Level-3 hub

Level-2 hub

Level-1 hub
Level-1 demand within the noncompetitive area $(0-50 \mathrm{~km})$

Level-1 demand within the competitive area $(50-200 \mathrm{~km})$
Level-2 demand within the noncompetitive area $(200-800 \mathrm{~km})$

Level-2 demand within the

competitive area $(800-1500 \mathrm{~km})$

Figure 3 - The optimal solution of the case study in scenario 3 and 4

optimal hub locations obtained for scenarios 3 and 4 by graphically solving the problem. In different scenarios, different solutions are obtained based on the allocation of demand nodes to the hub nodes. For brevity, we only present the allocation results for nodes 5 and 11 . We can conclude that the competition among hubs significantly affects the amount of the demand served by each hub. For example, in scenario 4 , in which only a level-1 hub at node 7 is operational, the level-1 demand within the noncompetitive area at node 5 (the red line in Figure 3) served by the level-1 hub at node 7 significantly increases compared with the case in scenario 3 , in which level-1 hubs are located at node 7 and node 6 , respectively. Geographical accessibility also plays a significant role in competition between transportation modes. The demand at node 11 can efficiently receive service from the level-1 hub at node 11 in

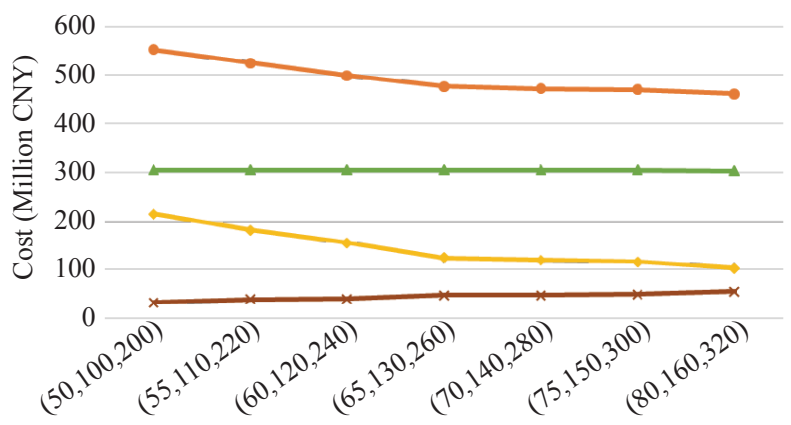

Maximum acceptable distances for service at each level $[\mathrm{km}]$ $\rightarrow \mathrm{TC} \rightarrow \mathrm{OC} \leftarrow \mathrm{TRC} * \mathrm{AC}$

a) Cost scenario 3. However, in scenario 4, a new level-1 hub at node 13 is operational, and the level-1 demand within the noncompetitive area serviced by node 11 (the green line in Figure 3) decreases.

\subsection{Sensitivity analysis}

In this subsection, we analyze the impact of changing the key parameters on the model results. All the experiments are conducted based on scenario 3 .

Figure 4 illustrates the effect of the maximum acceptable distance on the system cost and the average demand served by hubs at each level. When the maximum acceptable distance for services at each level increases, the established hubs can serve passengers from much farther locations. Thus, the access cost increases and the hub operation cost dramatically drops, leading to a reduction in the

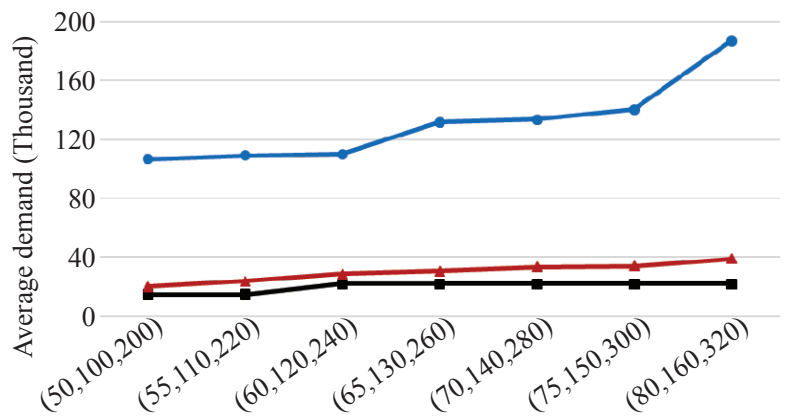

Maximum acceptable distances for service at each level [km] - Level-3 hubs - Level-2 hubs - Level-1 hubs

b) Average demand

Figure 4-Impact of the maximum acceptable distances on the system performance 
total cost. Moreover, the average demand served by passenger hubs at each level grows with increasing maximum acceptable distance. We can conclude an improvement in public transportation accessibility would increase the efficiency of transportation hubs. Therefore, to design an efficient passenger hub network in a megaregion area, it is crucial to consider improvements to hub accessibility.

The impact of $\delta_{i}$ on system performance is presented in Figure 5. To examine this influence, we prepared different VOT data sets varying from 0 to $140 \%$ of the original VOT data. As shown, when $\delta_{i}$ increases, the total cost gradually increases as the travel cost and access cost increase. However, the hub operation cost is relatively steady, which is mainly related to considering the maximum acceptable distance and limiting capacity in the model. Total travel time is one of the most important factors that affect hub choice behavior. More passengers choose the transportation with higher speed as the VOT increases. As a result, the average demand met by level-2 hubs takes an increasing trend while the average demand served by level-1 hubs decreases. This trend could have a significant impact on hub network design.

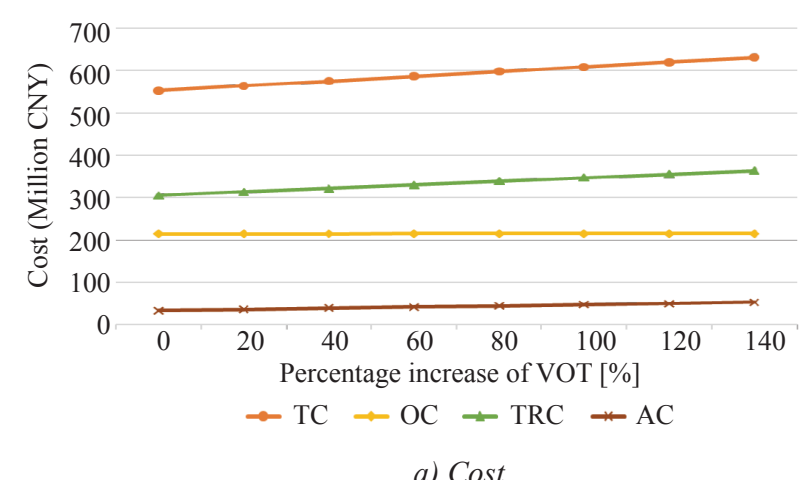

a) Cost

\subsection{Comparison of results without considering hub choice}

Unlike in the hub choice model, the demand nodes have to be allocated to the passenger hub nodes by system designers in the allocation step of the traditional HLPs. The multi-demand feature of this problem requires the allocation step to be treated as a mode choice problem in which passengers are assumed to choose between the available transportation services and the choice of mode leads to the use of one of the located passenger hubs. Therefore, it is essential to include the hub choice behavior in model formulation. To illustrate the significance of this consideration, a model that does not consider hub choice is tested. In this test, without loss of generality, Constraints 17 and 18 are removed. The comparative results are summarized in Table 3.

Table 3 shows that the traditional model that does not consider hub choice has no effect on the optimal hub location plan (row 6), but the allocation plan is different. More passengers are allocated to level-1 hubs, and the total demand served by level-2 hubs is generally less than that in the proposed model. Notably, there are fewer level-1

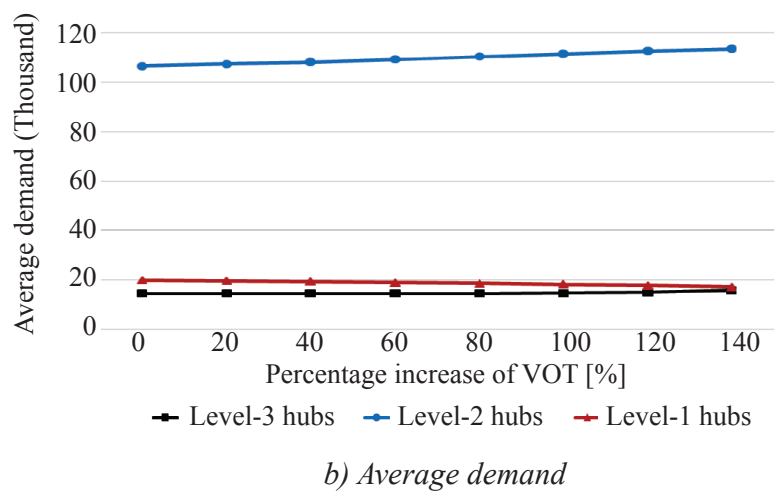

b) Average demand

Table 3 - Comparison of model results without considering hub choice

\begin{tabular}{||c|c|c|c|c|c||}
\hline \multicolumn{2}{|c|}{ Scenario number } & 1 & 2 & 3 & 4 \\
\hline \hline \multirow{3}{*}{$\begin{array}{l}\Delta \text { Total demand served by } \\
\text { level-k hubs [\%] }\end{array}$} & $k=1$ & 7.03 & 12.29 & 6.10 & 4.40 \\
\cline { 2 - 6 } & $k=2$ & -7.11 & -6.56 & -3.42 & -2.41 \\
\cline { 2 - 6 } & $k=3$ & 0.00 & 0.00 & 0.00 & 0.00 \\
\hline \multirow{4}{*}{\begin{tabular}{l} 
Cost [\%] \\
\cline { 2 - 6 }
\end{tabular}} & $\Delta \mathrm{TC}$ & -0.30 & -0.41 & -0.23 & -0.22 \\
\cline { 2 - 6 } & $\Delta \mathrm{OC}$ & 0.00 & 0.00 & 0.00 & 0.00 \\
\cline { 2 - 6 } & $\Delta \mathrm{TRC}$ & 0.13 & 0.26 & 0.04 & -3.03 \\
\cline { 2 - 6 } & $\Delta \mathrm{AC}$ & -5.26 & -7.91 & -4.32 & -3.76 \\
\hline
\end{tabular}


hubs than level-2 hubs, and passengers always receive services from the nearest passenger hub in the traditional model. Hence, in practice, the level-2 hubs would have more passengers because of the hub choice behavior. This difference in demand allocation may also lead to different passenger hub capacity decisions, which depend on the allocation results.

As shown in Table 3, the total cost of passengers (TRC and AC), especially the access cost, is less than that in the proposed model based on the same optimal passenger hub location plan. This finding suggests that the traditional model can produce a lower cost when each passenger is assigned to a hub by system designers. Therefore, it is advisable to guide passengers to choose the best passenger hub in practice, which can be achieved by the improvement of access transportation and the implementation of subsidy policy.

\section{CONCLUSIONS AND FUTURE RESEARCH}

In this paper, we propose a hierarchical passenger hub location model to assist in the design of passenger transportation networks in a megaregion area. The presented model considers a hierarchical passenger transportation network for multilevel travel demands with a new service availability type and applies a logit discrete-choice model to determine the proportion of passengers that access a hub depending on utility. We formulate the problem as a mixed integer linear programming problem to minimize the total cost of hub operation and transportation for multiple travel demands with hub capacity constraints. To evaluate the efficiency and effectiveness of the proposed model, it is implemented for the WHM area in four different scenarios. The results show that (1) the total demand served by passenger hubs at each level differs depending on the characteristics of the hierarchical passenger hub structure, (2) more intense competition has a positive impact on passengers due to decreases in the travel and access costs, and (3) establishing comprehensive transportation hubs could yield better economic benefits than designing hubs that only offer single transportation mode. A sensitivity analysis is performed to test the impact of changing key parameters on the model results. We find that the maximum acceptable distance for services at each level has a significant influence on system performance because it can increase the efficiency of transporta- tion hubs. Therefore, to design an efficient hub network in a megaregion area, it is crucial to consider improving passenger hub accessibility. In addition, the travel demand will shift to high-level hubs as the VOT increases. Differences in demand allocation are also found in all four scenarios when comparing the results with and without considering hub choice. This difference in allocation may also lead to different hub capacity decisions, which depend on the allocation results.

Future studies can be conducted to consider the effects of traffic congestion and the waiting time at a hub, which would require a stochastic hub location modelling framework. In addition, this paper considered a simple setting to demonstrate the proposed methodology. Although the hub operation cost and travel demand of each node may be estimated before planning, in practice, it is important to investigate situations with uncertainty related to the setup costs and travel demands. These topics require further research.

\section{ACKNOWLEDGEMENTS}

This study has been substantially supported by the National Natural Science Foundation of China through several projects (Grant Nos. 72021002, 71531011 and 71890973) and a project sponsored by the program of Shanghai academic research leader (No. 19XD1403800).

\section{闵黄, 博士生 ${ }^{1}$}

邮箱: tjyh@tongji.edu.cn

张小宁, 博士 ${ }^{1}$

（通讯作者）邮箱: cexzhang@tongji.edu.cn

王晓蕾, 博士 ${ }^{1}$

邮箱: xiaoleiwang@tongji.edu.cn

1 同济大学经济与管理学院, 中国上海市四平路 1239 号

考虑服务可得性的城市群客运枢纽层级选址问题

摘要

城际出行需求的快速增长对城市群客运交通网络 造成了极大压力. 而通过优化枢纽选址和需求分配 可以提高客运交通网络的运行效率. 本文考虑层级 枢纽的服务可得性特征, 构建了城市群客运枢纽层 级选址模型. 以枢纽运营费用和层级交通需求的运 输费用最小化为目标, 求解混合整数规划模型确定 分配到各级枢纽的乘客比例. 以四个不同场景下武 汉城市群的算例验证了模型的适用性. 其次, 利用 敏感性分析评估关键参数对模型结果的影响. 最后, 与传统模型结果对比发现: 在需求分配阶段考虑枢 纽选择行为具有重要意义. 


\section{关键词}

客运枢纽层级选址；城市群；服务可得性； 枢纽选择行为

\section{REFERENCES}

[1] Wang J, Huang J, Jing Y. Competition between highspeed trains and air travel in China: From a spatial to spatiotemporal perspective. Transportation Research Part A: Policy and Practice. 2020;133: 62-78. DOI: 10.1016/j.tra.2019.12.030

[2] Li H, Wang K, Yu K, et al. Are conventional train passengers underserved after entry of high-speed rail?-evidence from Chinese intercity markets. Transport Policy. 2020;95: 1-9. DOI: 10.1016/j.tranpol.2020.05.017

[3] D'Alfonso T, Jiang C, Bracaglia V. Would competition between air transport and high-speed rail benefit environment and social welfare?. Transportation Research Part B: Methodological. 2015;74: 118-137. DOI: 10.1016/ j.trb.2015.01.007

[4] Pašagić Škrinjar J, Rogić K, Stanković R. Location of Urban Logistic Terminals as Hub Location Problem. Promet - Traffic\&Transportation. 2012;24(5): 433-440. DOI: $10.7307 /$ ptt.v24i5.1179

[5] Kim J-H, Soh S. Designing Hub-and-Spoke School Bus Transportation Network: A Case Study of Wonkwang University. Promet - Traffic\&Transportation. 2012;24(5): 389-394. DOI: 10.7307/ptt.v24i5.1174

[6] Šarac D, Kopić M, Mostarac K, Kujačić M, Jovanović B. Application of Set Covering Location Problem for Organizing the Public Postal Network. Promet - Traffic\&Transportation. 2016;28(4): 403-413. DOI: 10.7307/ ptt.v28i4.1962

[7] O'Kelly ME. The Location of Interacting Hub Facilities. Transportation Science. 1986;20(2): 92-106. DOI: 10.1287/trsc.20.2.92

[8] O`Kelly ME. A Quadratic Integer Program for the Location of Interacting Hub Facilities. European Journal of Operational Research. 1987;32(3): 393-404. DOI: 10.1016/S0377-2217(87)80007-3

[9] Campbell JF. Hub Location and the p-hub Median Problem. Operations Research. 1996;44(6): 923-935. DOI: 10.1287/opre.44.6.923

[10] Campbell JF. Integer Programming Formulations of Discrete Hub Location Problem. European Journal of Operational Research. 1994;72(2): 387-405. DOI: 10.1016/0377-2217(94)90318-2

[11] Costa MG, Captivo ME, Clímaco J. Capacitated Single Allocation Hub Location Problem-A Bi-criteria Approach. Computers \& Operations Research. 2008;35(11): 3671-3695. DOI: 10.1016/j.cor.2007.04.005

[12] Farahani RZ, Hekmatfar M, Arabani AB, Nikbakhsh E. Hub Location Problems: A Review of Models, Classification, Solution Techniques, and Applications. Computers Industrial Engineering. 2013;64(4): 1096-1109. DOI: $10.1016 /$ j.cie.2013.01.012

[13] Flynn J, Ratick S. A Multiobjective Hierarchical Covering
Model for the Essential Air Services Program. Transportation Science. 1988;22(2): 139-147. DOI: 10.1287/ trsc.22.2.139

[14] Current JR. The Design of a Hierarchical Transportation Network with Transshipment Facilities. Transportation Science. 1988;22(4): 270-277. DOI: 10.1287/trsc.22.4.270

[15] O`Kelly ME, Lao Y. Mode Choice in a Hub-and-Spoke Network: A Zero-One Linear Programming Approach. Geographical Analysis. 1991;23(4): 283-297. DOI: 10.1111/j.1538-4632.1991.tb00240.x

[16] Arnold P, Peeters D, Thomas I. Modelling a Rail/Road Intermodal Transportation System. Transportation Research Part E: Logistics and Transportation Review. 2004;40(3): 255-270. DOI: 10.1016/j.tre.2003.08.005

[17] Yu J, Liu Y, Chang GL, Ma WJ, Yang XG. Cluster-Based Hierarchical Model for Urban Transit Hub Location Planning: Formulation, Solution, and Case Study. Transportation Research Record. 2009;2112(1): 8-16. DOI: $10.3141 / 2112-02$

[18] Narula SC. Hierarchical Location-Allocation Problems: A Classification Scheme. European Journal of Operational Research. 1984;15(1): 93-99. DOI: 10.1016/03772217(84)90052-3

[19] Karimi M, Eydi AR, Korani E. Modeling of the Capacitated Single Allocation Hub Location Problem with a Hierarchical Approach. International Journal of Engineering. 2014;27(4): 573-586. DOI: 10.5829/idosi. ije.2014.27.04a.08

[20] Li TT, Song R, He SW, Bi MK, Yin WC, Zhang YQ. Multiperiod Hierarchical Location Problem of Transit Hub in Urban Agglomeration Area. Mathematical Problems in Engineering. 2017. DOI: 10.1155/2017/7189060

[21] Torkestani SS, Seyedhosseini SM, Makui A, Shahanaghi K. The Reliable Design of a Hierarchical Multi-modes Transportation Hub Location Problems (HMMTHLP) under Dynamic Network Disruption (DND). Computers Industrial Engineering. 2018;122: 39-86. DOI: 10.1016/j.cie.2018.05.027

[22] Debrezion G, Pels E, Rietveld P. Modelling the Joint Access Mode and Railway Station Choice. Transportation Research Part E: Logistics and Transportation Review. 2009;45(1): 270-283. DOI: 10.1016/j.tre.2008.07.001

[23] Wang S, Qu X. Station Choice for Australian Commuter Rail Lines: Equilibrium and Optimal Fare Design. European Journal of Operational Research. 2017;258(1): 144-154. DOI: 10.1016/j.ejor.2016.08.040

[24] Aros-Vera F, Marianov V, Mitchell JE. P-hub Approach for the Optimal Park-and-Ride Facility Location Problem. European Journal of Operational Research. 2013;226(2): 277-285. DOI: 10.1016/j.ejor.2012.11.006

[25] Lofberg J. YALMIP: A Toolbox for Modeling and optimization in MATLAB. In: Proceedings of 2004 IEEE International Conference on Robotics and Automation. New Orleans, LA; 2004. p. 284-289.

[26] Huang HJ, Xia T, Tian Q, Liu TL, Wang C, Li D. Transportation Issues in Developing China's Urban Agglomerations. Transport Policy. 2020;85: A1-A22. DOI: $10.1016 /$ j.tranpol.2019.09.007 